\title{
The Recurrent Eclipsing Nova U Sco: A Short Review
}

\author{
R. González-Riestra ${ }^{1}$ \\ ${ }^{1}$ XMM Science Operations Centre, ESAC, Madrid, Spain \\ Corresponding author: Rosario.Gonzalez@sciops.esa.int
}

\begin{abstract}
U Scorpii is the recurrent nova with the shortest inter-outburst period, only ten years. The last active phase took place at the beginning of 2010, and it provided a large amount of data from both ground-based and space observatories. This paper reviews some of the more relevant recent findings and points out some, still unanswered, questions.
\end{abstract}

Keywords: recurrent novae - UV - X-rays - individual: U Sco.

\section{Introduction}

$\mathrm{U}$ Scorpii is a recurrent nova that undergoes frequent outbursts. It is also an eclipsing binary $(\mathrm{i}=83 \mathrm{deg}$, Thoroughgood et al. 2001), hence the geometrical parameters of the system are known with great accuracy. The orbital period is 1.23 days (Schaefer 1990).

Ten outbursts of this system have been observed, the first one in 1863 (Schaefer 2010). Outbursts take place quite regularly, every $\approx 10$ years. There are two long intervals of about 20 years during which no outbursts were observed (around 1927 and 1957), but very likely they were missed because the system was too close to the Sun when they took place.

The system is characterised by extremely short timescales: the recurrence time is only ten years, the shortest known in this type of objects, the system goes into outburst in a few hours, and decays in a few days, with $t_{2}=1.2$ days and $t_{3}=2.6$ days. In accordance with all this, very high velocities of the order of $10^{4} \mathrm{~km} \mathrm{~s}^{-1}$ have been observed.

All the above implies that the primary of the system is a high-mass white dwarf. The nature of the secondary star is still unclear, but it is very likely slightly evolved, K2 IV (Anupama and Dewangan 2000). There is a general agreement on the sizes and masses of both stars: $\mathrm{R}_{2} \approx 2.4 \mathrm{R}_{\odot}, \mathrm{M}_{2} \approx 1.3 \mathrm{M}_{\odot}, \mathrm{M}_{1} \geq 1.37 \mathrm{M}_{\odot}$ (Hachisu et al. 2000, Thoroughgood et al. 2001). The size of the orbit is $\approx 6.7 \mathrm{R}_{\odot}$, and the distance to the system has been estimated to be $12 \mathrm{kpc}$ (Schaefer 2010).

\subsection{Before 2010: UV data}

IUE observed U Sco during the first weeks of the 1979 outburst. The UV spectrum presented at the beginning low ionisation lines, and strong P-Cygni profiles in the resonance lines. Later on, there was an overall increase in the ionisation level, so that in the last observations

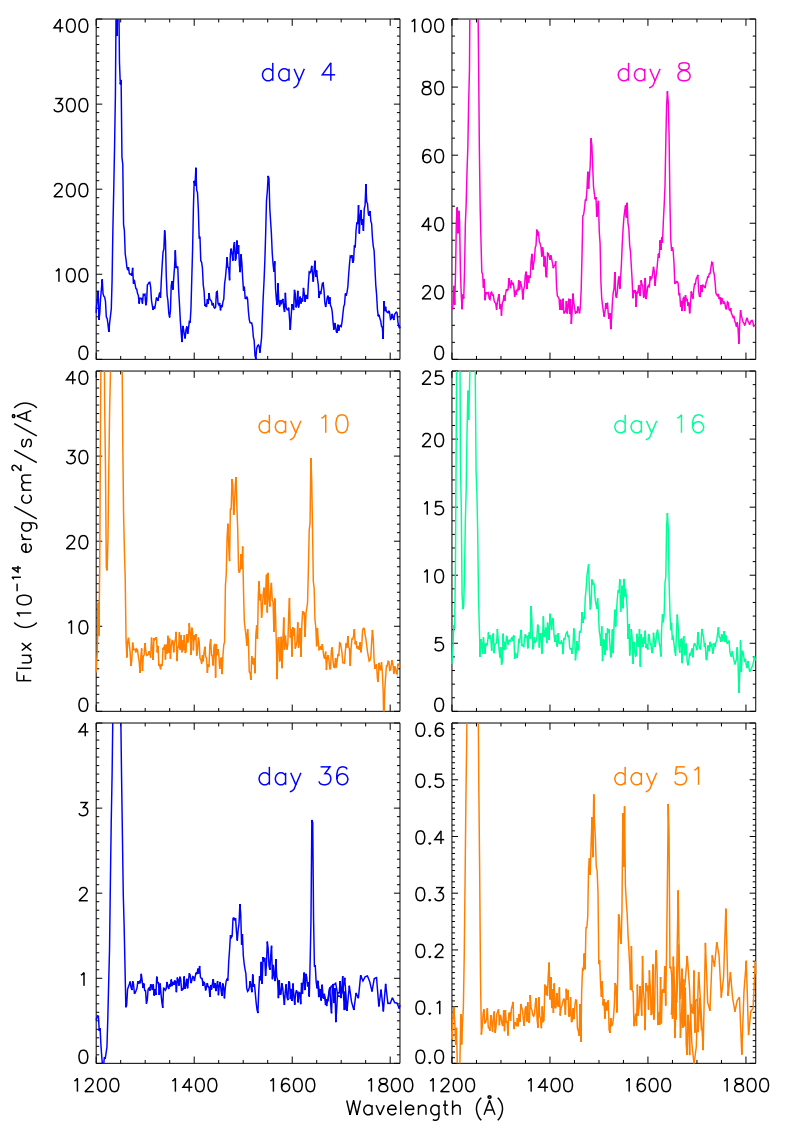

Figure 1: Evolution of the ultraviolet spectra of U Sco during active phases. Spectra of days 4-16 were taken with IUE during the 1979 outburst, while the last two were taken with HST-STIS in 1999. 
The Recurrent Eclipsing Nova U Sco: A Short Review

the strongest lines corresponded to He II, N V, C IV, N IV, and possibly [Mg V] (Williams et al. 1981, Barlow et al. 1981).

It is worth noting that the He II $1640 \AA$ line was always much narrower than the others.

Further UV observations were obtained with HSTSTIS during the first two months of the 1999 outburst, confirming the evolution observed before (Fig. 1). The last spectra showed only high ionisation broad lines and a narrow He II line.

\subsection{Before 2010: X-ray data}

U Sco was known to be a strong X-ray emitter already before the 2010 outburst. The SAX observation on day 19 of the 1999 outburst, when the system was detected as a supersoft source, is described by Kahabka et al. (1999). These authors fitted a model atmosphere to the SAX spectrum, finding a temperature of $9 \times 10^{5} \mathrm{~K}$ and a luminosity in the range $2-20 \times 10^{37} \mathrm{erg} \mathrm{s}^{-1}$. They also found evidence for a weak harder component, and reported variations of the order of $50 \%$ during the observation.

\section{The 2010 Outburst}

By the beginning of the $21^{\text {st }}$ century the recurrence time of U Sco was well established, $10 \pm 2$ years (Schaefer 2005). The last outburst had taken place in 1999, and the date of the next one was predicted to be $2009.3 \pm 1$. In very good agreement with this prediction, the outburst took place on January 282010 (2010.08). The time to rise to maximum brightness was only 9 hours. The optical light curve was very complex, with many different features: there was an early decline phase, a plateau, a second decline and a second plateau. And superimposed on all this, there were short-lived phenomena: flares, dips and the resumption of eclipses (see a detailed description in Schaefer et al. 2011).

\subsection{X-ray observations}

\subsubsection{Swift}

The 2010 outburst of U Sco was followed by all the X-ray missions, Swift, Suzaku, Chandra and XMMNewton.

Particularly relevant is the monitoring carried out by Swift since, apart from its own scientific value, it allowed to schedule efficiently Target of Opportunity observations with the other missions, as described below.

Swift observed U Sco daily during two months. Supersoft X-ray emission was detected around day 12, when the flux started to increase until reaching a maximum around day 31 , to decrease slowly afterwards. The appearance of the supersoft emission roughly coincided with the first extended optical plateau, while the X-ray turn-off started at the time of the second optical decline (Schaefer et al. 2010).

\subsubsection{Suzaku}

Suzaku took three observations of U Sco, but only the last one, on day 15, provided useful data, and a surprising result: the detection of a shallow X-ray eclipse (with a depth of $\approx 30 \%$ ) in coincidence with the predicted time of the optical eclipse. From the duration of this eclipse, Takei et al. (2013) estimated the size of the X-ray emitting region in $5 \mathrm{R}_{\odot}$.

\subsubsection{High resolution spectroscopy: Chandra and XMM-Newton}

A further X-ray observation was taken on day 18, this time with Chandra LETG. Unfortunately, it was short, and taken out of eclipse, and therefore the Suzaku result could not been confirmed. Orio et al. (2013) claimed that the deep absorption lines seen in the LETG spectrum (see top panel of Fig. 2) are not P-Cygni profiles, but just the superposition of blue-shifted absorptions from the white dwarf atmosphere (whose shift is due to a wind) and the emission lines from the ejecta. These authors derived a temperature of $7 \times 10^{5} \mathrm{~K}$ and a luminosity of $7 \times 10^{36} \mathrm{erg} \mathrm{s}^{-1}$. This is to be compared with the SAX estimations, with a much higher luminosity. It is not clear whether the 1999 outburst was intrinsically more luminous, or if the discrepancy is just due to the different atmosphere models used for both determinations.

Two more X-ray high resolution spectra were obtained with XMM-Newton on days 23 and 35, the second one just after the X-ray peak. The XMM-RGS spectrum of the first observation showed similar emission lines as the Chandra spectrum, but no absorptions. This was interpreted by Orio et al. (2013) as an indication of the end of the wind phase of the white dwarf. The model atmosphere fits presented by these authors showed an increase in the temperature of the white dwarf from day 18 to day 35 , at a a nearly constant X-ray luminosity. The emission lines in the day 23 spectrum can be explained by collisional ionisation, while the situation is more complex on day 35 , requiring also the existence of a strong UV field. There are some unidentified lines, and others whose presence just cannot be explained. The three grating spectra are shown in left panel of Fig. 2.

The X-ray light curves also provided interesting information. The first XMM observation started at orbital phase 0.8 , thus a clear X-ray eclipse was expected, but an irregular light curve was seen instead, with dips and flares. These dips are an indication of clumps of 
material in the re-forming accretion disk (Ness et al. 2012). On the contrary, on day 35 the eclipse is very well defined. It is worth to note that on this date the depth of the eclipse is $50 \%$, showing that the $\mathrm{X}$-ray source is very extended, likely a Thomsom scattering corona (Ness et al. 2012, Orio et al. 2013).

Takei et al. (2013) interpreted the difference in the $\mathrm{X}$-ray eclipses of days 15 and 35 as a shrinking of the $\mathrm{X}$-ray emitting region, from 5 to $4 \mathrm{R}_{\odot}$. The optical eclipse mapping done by Schaefer et al. (2011) also indicates a shrinking of the optical source, from $4 \mathrm{R}_{\odot}$ on day 20 to $2.2 \mathrm{R}_{\odot}$ on day 50 . After day 20 , the source of the optical light changes, from spherically symmetric to disk-like, first with a bright rim, later brighter at the centre.

XMM-Newton obtained UV photometry simultaneously with the X-ray observations. On day 23 there was a very well defined UV eclipse, and the light curve was rather smooth, with some structures, as a flare that can be coincident with a similar feature in the X-ray light curve. The depth of the minima in both UV and X-ray light curves was similar $(\approx 50 \%)$. The eclipse on day 35 is twice as deep in the UV than in X-rays, indicating a smaller emitting area (Ness et al. 2012).

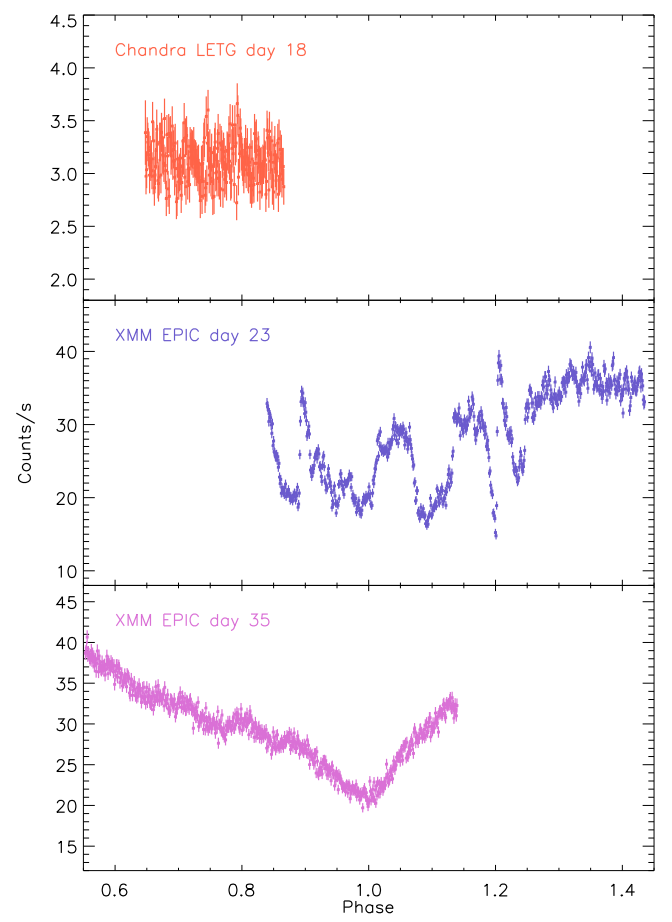

Figure 2: X-ray high resolution spectra (left panel) and light curves (right panel) of U Sco during the 2010 outburst. From top to bottom, Chandra LETG data taken on day 18, and XMM-Newton RGS spectra and EPIC light curves obtained on days 23 and 35.

\subsection{Optical spectroscopy}

Also interesting to mention is the evolution of the optical spectra. Mason et al. (2012) reported the appearance of narrow emission lines of $\mathrm{H}$, He I and He II on day 8 . The radial velocity curve of these lines (that does not mach either of the two stars) indicate that they form in an accretion flow suggesting that the disk was already re-established.

Mason (2011) studied the abundance in the ejecta and found a high $\mathrm{Ne} / \mathrm{O}$ ratio, concluding that the white dwarf belongs to the O-Ne-Mg class, with the implications for the further evolution of the system.
Finally, in this outburst, forbidden emission lines have been observed for the first time, around day 46 (Mason et al. 2012, Díaz et al. 2010). This result must be taken with caution, as it can be just a selection effect, since this outburst had a much better observational coverage than the previous ones.

\subsection{The evolution of the outburst}

As shown in the previous sections, the last outburst of U Scorpii has provided a large amount of data that have given us to a more clear view of the behaviour of the system. 
Models show he disk was destroyed in the outburst (Drake and Orlando 2010). But only a few days later there were already evidences of accretion being re-established, e.g.:

- appearance of narrow optical lines on day 8 ,

- optical and X-ray eclipses on day 15, indicating the existence of a large emitting region $\approx 4-5 \mathrm{R}_{\odot}$,

- optical flickering and dips in the X-ray light curve on day 23.

Analysis of the optical light curve shows that by day 40 the disk was already fully re-established.

Table 1: Summary of the evolution of the 2010 outburst of U Scorpii

\begin{tabular}{ll}
\hline Day & Event \\
\hline 0 & Disk destroyed \\
8 & He, HeI and HeII narrow emission lines \\
$9-15$ & Optical flares \\
12 & Supersoft phase \\
15 & X-ray and optical eclipses \\
20 & End of the white dwarf mass-loss \\
23 & X-ray dips \\
24 & Flickering \\
30 & Rim-bright disk \\
40 & Optical dips \\
46 & Nebular spectrum \\
\hline
\end{tabular}

\section{Conclusions}

This system represents an unique opportunity to study the outburst mechanisms of recurrent novae, as it allows us to compare data obtained in several different cycles, with relatively similar instrumentation. Despite of the large amount of available data, or possibly due to that, there are still many open issues. For instance, when going through the literature, the values of the mass of the envelope cover a range of 60 , even for the same outburst. Williams at al. (1981) and Anupama and Dewangan (2000) get the same value of $10^{-7} \mathrm{M}_{\odot}$ for the 1979 and the 1989 outbursts, respectively. On the other hand, Takei et al. (2013) obtain $6 \times 10^{-6} \mathrm{M}_{\odot}$ for the last outburst. Also striking is the wide range of Helium abundance values $(\mathrm{N}(\mathrm{He}) / \mathrm{N}(\mathrm{H}))$, as high as 4.5 (Evans et al. 2001), but also as low 0.07 (Maxwell et al. 2012). Is there is a real difference in the mass ejected in the different outbursts? How can the values of the Helium abundance be reconciled?

Obviously, the methods used to derive this quantities and the underlying assumptions are very different. A deep, uniform analysis of all existing data is necessary to find the reason for these discrepancies and to better understand the behaviour of the system.

\section{Acknowledgement}

I warmly thank the organisers for their kind invitation to this Meeting.

\section{References}

[1] Anupama, G. and Dewangan, G.: 2000, AJ 119, 1359

[2] Barlow, M., Brodie, J., Brunt, C. et al.: 1981, MNRAS 195, 61

[3] Díaz, M., Williams, R., Luna, G. et al.: 2010, AJ 140,1860

[4] Drake, J. and Orlando, S.: 2010: ApJ, 720, L195 doi:10.1088/2041-8205/720/2/L195

[5] Evans, A., Krautter, J., Vanzi, L. and Starrfield, S.: 2001, A\&A 378, 132

[6] Hachisu, I.; Kato, M., Kato, T. and Matsumoro, K.: 2000, ApJ 534, L189

[7] Kahabka, P., Hartmann, H., Parmar, A. and Negueruela, I.: 1999. A\&A 347, 43

[8] Mason, E., 2011, A\&A 532, L11

[9] Mason, E., Ederoclite, A., Williams, R. et al.: 2012, A\&A 544, 149

[10] Maxwell, M., Rushton, M., Darnley, M. et al.: 2012, MNRAS 419, 1465 doi:10.1111/j.1365-2966.2011.19803.x

[11] Ness, J.-U., Schaefer, B., Dobrotka, A. et al.: 2012. ApJ 745, 43 doi:10.1088/0004-637X/745/1/43

[12] Orio, M., Behar, E., Gallagher, J. et al.: 2013, MNRAS 429, 1342 doi:10.1093/mnras/sts421

[13] Takei, D., Drake, J., Tsujimoto, M. et al.: 2013, ApJ 769, L4 doi:10.1088/2041-8205/769/1/L4

[14] Schaefer, B.: 1990, ApJ 355, L39

[15] Schaefer, B.: 2005, ApJ, 621, L53 doi:10.1086/429145 
[16] Schaefer, B.: 2010, ApJS 197, 275 doi:10.1088/0067-0049/187/2/275

[17] Schaefer, B., Pagnotta, A., Osborne, J. et al.: 2010, ATEL 2477

[18] Schaefer, B., Pagnotta, A., LaCluyze, A. et al.: 2011, ApJ 742, 113
doi:10.1088/0004-637X/742/2/113

[19] Thoroughgood, T., Dhillon, V., Littlefair, S. et al.: 2001, MNRAS 327, 1323

[20] Williams, R., Sparks, W. Gallagher, J. et al.: 1981, ApJ 251, 221 